\title{
Quasi-simultaneous Radio/X-Ray Observations of the Candidate Transitional Millisecond Pulsar 3FGL J1544.6-1125 during its Low-luminosity Accretion-disk State
}

\author{
Amruta D. Jaodand ${ }^{1,2,3}$ (1) , Adam T. Deller ${ }^{4}$ (1), Nina Gusinskaia, ${ }^{3,5}$, Jason W. T. Hessels ${ }^{2,3}$ (i), James C. A. Miller-Jones ${ }^{6}$ (i)

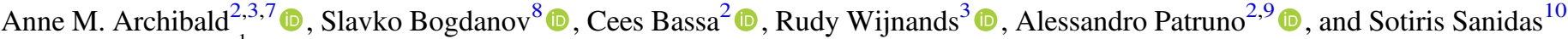 \\ ${ }^{1}$ California Institute of Technology, 1200 E California Blvd., Pasadena, CA 91125, USA; ajaodand@caltech.edu \\ 2 ASTRON, The Netherlands Institute for Radio Astronomy, Postbus 2, 7990 AA Dwingeloo, The Netherlands \\ ${ }^{3}$ Astronomical Institute Anton Pannekoek, University of Amsterdam, 1098XH, Amsterdam, The Netherlands \\ ${ }^{4}$ Centre for Astrophysics and Supercomputing, Swinburne University of Technology, Mail Number H74, PO Box 218, Hawthorn, VIC 3122, Australia \\ ${ }^{5}$ David D Dunlap Institute of Astronomy and Astrophysics, University of Toronto, Ontario, M5S 3H4, Canada \\ ${ }^{6}$ International Centre for Radio Astronomy Research-Curtin University, GPO Box U1987, Perth, WA 6845, Australia \\ ${ }^{7}$ School of Mathematics, Statistics, and Physics, Newcastle University, Newcastle upon Tyne NE1 7RU, UK \\ ${ }^{8}$ Columbia Astrophysics Laboratory, Columbia University, 550 West 120th Street, New York, NY 10027, USA \\ ${ }^{9}$ Institute of Space Sciences (IEEC-CSIC) Campus UAB, Carrer de Can Magrans, s/n, E-08193 Barcelona, Spain \\ ${ }^{10}$ Jodrell Bank Centre for Astrophysics, School of Physics and Astronomy, The University of Manchester, Manchester M13 9PL, UK \\ Received 2020 December 23; revised 2021 August 20; accepted 2021 August 21; published 2021 December 7
}

\begin{abstract}
3FGL J1544.6-1125 is a candidate transitional millisecond pulsar (tMSP). Similar to the well-established tMSPs-PSR J1023+0038, IGR J18245-2452, and XSS J12270-4859-3FGL J1544.6-1125 shows $\gamma$-ray emission and discrete X-ray "low" and "high" modes during its low-luminosity accretion-disk state. Coordinated radio/X-ray observations of PSR J1023+0038 in its current low-luminosity accretion-disk state showed rapidly variable radio continuum emission-possibly originating from a compact, self-absorbed jet, the "propellering" of accretion material, and/or pulsar moding. 3FGL J1544.6-1125 is currently the only other (candidate) tMSP system in this state, and can be studied to see whether tMSPs are typically radio-loud compared to other neutron star binaries. In this work, we present a quasi-simultaneous Very Large Array and Swift radio/X-ray campaign on 3FGL J1544.6-1125. We detect $10 \mathrm{GHz}$ radio emission varying in flux density from $47.7 \pm 6.0 \mu \mathrm{Jy}$ down to $\lesssim 15 \mu \mathrm{Jy}$ ( $3 \sigma$ upper limit) at four epochs spanning three weeks. At the brightest epoch, the radio luminosity is $L_{5 \mathrm{GHz}}=(2.17 \pm 0.17) \times 10^{27} \mathrm{erg} \mathrm{s}^{-1}$ for a quasi-simultaneous X-ray luminosity $L_{2-10 \mathrm{keV}}=(4.32 \pm 0.23) \times 10^{33} \mathrm{erg} \mathrm{s}^{-1}$ (for an assumed distance of $3.8 \mathrm{kpc}$ ). These luminosities are close to those of PSR J1023+0038, and the results strengthen the case that 3FGL J1544.6-1125 is a tMSP showing similar phenomenology to PSR J1023+0038.
\end{abstract}

Unified Astronomy Thesaurus concepts: Millisecond pulsars (1062); Binary pulsars (153); Radio continuum emission (1340); Accretion (14)

\section{Introduction}

Transitional millisecond pulsars (tMSPs) are a recently discovered type of binary system that transitions between rotation-powered radio millisecond pulsar (RMSP) and accretion-powered low-mass X-ray binary (LMXB) states (e.g., Archibald et al. 2009; Papitto et al. 2013; Bassa et al. 2014; Stappers et al. 2014; Roy et al. 2015). Currently, there are three well-established tMSP systems-PSR J1023+0038, IGR J18245-2452, and XSS J12270-4859-and three excellent candidate transitional millisecond pulsar systems3FGL J1544.6-1125, CXOU J110926.4-650224 (Coti Zelati et al. 2019), and 4FGL J0427.8-6704 (Strader et al. 2016; Kennedy et al. 2020).

To conclusively classify a neutron star (NS) binary as a tMSP, one needs to demonstrate that the system has been observed in both the rotation-powered RMSP and accretionpowered LMXB states. Nonetheless, in the absence of an observed transition, we can still make a strong case that a particular candidate system is of a similar ilk to the tMSPs and may in the future undergo a state transition. Fortunately, the tMSPs provide a rich observational phenomenology that helps build such a connection (see Table 2), with the caveat that there are only three such systems currently known and perhaps a larger sample will display a larger diversity.
In their $\mathrm{LMXB}^{11}$ state, tMSPs show distinct X-ray luminosity modes. For example, PSR J1023+0038 switches within $\lesssim 10$ s between "high" $\left(L_{2-10 \mathrm{keV}} \sim 10^{33} \mathrm{erg} \mathrm{s}^{-1}\right.$, present $\sim 80 \%$ of the time) and "low" $\left(L_{2-10 \mathrm{keV}} \sim 10^{32} \mathrm{erg} \mathrm{s}^{-1}\right.$, present $\sim 20 \%$ of the time) X-ray luminosity modes (Archibald et al. 2015; Bogdanov et al. 2015; Jaodand et al. 2016). These modes last typically for minutes up to an hour. Occasionally, there are instances of X-ray "flares" $\left(L_{2-10 \mathrm{keV}} \sim 10^{34} \mathrm{erg} \mathrm{s}^{-1}\right)$. A very similar variability pattern was observed in XSS J12270-4859, prior to its 2012 transformation to an RMSP (de Martino et al. 2013), whereas IGR J18245-2452's moding behavior differs as it shows longer-lasting modes (several hours' duration; see Linares et al. 2014).

Coherent X-ray pulsations are observed in PSR J1023+0038 and XSS J12270-4859 at the neutron star's rotational period during only the X-ray high mode (Archibald et al. 2015; Bogdanov et al. 2015; Papitto et al. 2015, 2019; Jaodand et al. 2016). Coherent pulsations at X-ray luminosities, typically considered as a "quiescent state" for other X-ray binaries, are surprising since the accretion material is unlikely to provide

\footnotetext{
11 In this paper we use the term "LMXB state" to distinguish from when a tMSP is observed as an RMSP, but caution that only one confirmed tMSP has been observed in a full LMXB outburst, whereas the other two have only been seen in a low-luminosity, persistent accretion-disk state that is unlike what has been observed to date in classical LMXBs.
} 
enough pressure to overcome the neutron star's magnetic pressure and to produce hot spots on the stellar surface-as are believed to power coherent pulsations from accreting X-ray millisecond pulsars (AMXPs). The effect has thus far only been observed in tMSPs, and could represent some exotic form of stimulated pulsar moding (Archibald et al. 2015). This moding behavior now extends to optical wavelengths (Papitto et al. 2019).

PSR J1023+0038 has been monitored across the electromagnetic spectrum since its transition to the LMXB state in 2013 (Patruno et al. 2014; Stappers et al. 2014). In the LMXB state, PSR J1023+0038 is the best studied tMSP system. Hence, we use it hereafter as the canonical system to outline phenomenological similarities in the tMSP class.

To better understand the puzzling low-luminosity tMSP accretion regime, we conducted a timing campaign on PSR J1023+0038 using XMM-Newton (Jaodand et al. 2016). In this two-year-long campaign we timed the coherent X-ray pulsations and found that the system is spinning down $(26.8 \pm 0.4) \%$ faster than in the RMSP state (i.e., compared to the radio-derived timing solution of Archibald et al. 2013). This measurement shows that the accretion torques have (on average) been roughly constant over $2 \mathrm{yr}$. Moreover, the lack of large torque variations, coupled with the spin-down rate being only $\sim 27 \%$ faster than in the RMSP state, tells us that the dipole spin-down mechanism observed in the RMSP state is still dominant and the pulsar wind continues to operate in a mostly unchanged manner. Enhanced spin-down could in principle be due to an increased proportion of open field lines in the pulsar magnetosphere (Parfrey et al. 2016, 2017) or to outflow of accretion material.

Additional insights come from radio continuum imaging of PSR J1023+0038 using the Karl G. Jansky Very Large Array (VLA). Deller et al. (2015) found approximately flat-spectrum $(-0.3<\alpha<0.3$, where the radio flux density $S$ scales with the observing frequency $\nu$ as $S \propto \nu^{\alpha}$ ) continuum radio emission that was observed to vary by up to an order of magnitude in brightness on timescales of minutes to an hour. This suggested partially self-absorbed synchrotron emission from a jet-like collimated outflow. The radio emission, which persisted for the span of the six-month observing campaign, was surprisingly bright given the low X-ray luminosity and inferred accretion rate. This was attributed to radiatively inefficient "propellermode" accretion, where there is ejection of inflowing accretion material because it is unable to overcome the centrifugal barrier at the corotation radius (where accretion material corotates with the neutron star's magnetic field). In such a situation, the jetlike outflow would then carry away the majority of the liberated accretion energy.

The tMSPs hence accrete at unexpectedly low X-ray luminosity levels (Archibald et al. 2015) and show higher radio luminosities than expected for other NS-LMXBs with millisecond spin rates (Deller et al. 2015). For example, Z-sources $^{12}$ are highly luminous as they accrete with nearEddington luminosities $\left(\geqslant 10^{38} \mathrm{erg} \mathrm{s}^{-1}\right)$, and their outbursts span timescales from a few hours to days. Atoll-sources ${ }^{13}$ are usually found to be accreting at luminosities $\leqslant 10^{38} \mathrm{erg} \mathrm{s}^{-1}$ on

\footnotetext{
12 They trace a Z-shape comprising flaring, normal, and horizontal branches from harder to softer colors in the hardness-intensity diagram (HID).

${ }^{13}$ Their HID traces show steady hardness over a range of intensities resembling small clusters or "islands" (see Hasinger \& van der Klis 1989; Muno et al. 2002; Church et al. 2014).
}

month-long timescales. Finally, rapid bursters are peculiar neutron star binaries that show a Type-I X-ray burst at the highest peak of outburst and the rare, intense Type-II X-ray bursts $^{14}$ erratically during periods of lower X-ray activity. The stable nature of the long-lasting (multiyear) accretion regime and abundance of information now available from multiwavelength campaigns (e.g., Bogdanov et al. 2015) has established tMSPs as excellent laboratories in which to study accretion onto magnetized neutron stars, at lower accretion rates than probed in previous studies.

Most recently, Bogdanov et al. (2018) uncovered a striking radio/X-ray anticorrelation in PSR J1023+0038 using strictly simultaneous Chandra X-ray Observatory and VLA observations. They found that all the X-ray "low" modes were reliably accompanied by simultaneous radio flux brightening, whose duration was closely matched to that of the low mode. Additional, sporadic radio flares-unassociated with X-ray low/high-mode switches, and only sometimes associated with X-ray flares-were also occasionally seen. During the X-ray low mode, the radio spectral index was observed to change from significantly inverted $(\alpha \simeq 0.4$ ) during the rise in the radio flare to relatively steep $(\alpha \simeq-0.5)$ during its decay. Going beyond the initial insights from the Deller et al. (2015) radio/ X-ray campaign, Bogdanov et al. (2018) concluded that the observations could not be explained by a canonical inflow/ outflow model, in which the radiated emission and the jet luminosity are powered by, and positively correlated with, the available accretion energy. They argued instead for a picture in which the X-ray high modes (with accompanying coherent $\mathrm{X}$-ray pulsations) represent active accretion into the neutron star magnetosphere (as also concluded by Archibald et al. 2015), whereas the X-ray low modes and associated radio flares represent the rapid ejection of plasma by the active rotationpowered pulsar-possibly initiated by a reconfiguration of the neutron star magnetosphere to another quasi-stable state. The only slightly enhanced neutron star spin-down rate found by Jaodand et al. (2016) appears consistent with this picture, in which the rotation-powered pulsar wind continues to play a significant role in shaping the multiwavelength emission properties of the system.

The cumulative observational findings achieved in the last decade of tMSP studies (Jaodand et al. 2017; Campana \& Di Salvo 2018; Papitto \& de Martino 2020) have given various insights into low-level accretion onto rapidly-spinning, highly magnetized neutron stars. We recap these observational findings for various sources in Table 1. The comprehensive picture of this accretion state, however, necessitates an increase in the population of known tMSPs, especially since two of the three known systems (XSS J12270-4859 and IGR J18245 -2452) are currently in the RMSP state.

\section{1. $3 F G L J 1544.6-1125$}

A very promising candidate tMSP, 3FGL J1544.6-1125, was recently found by Bogdanov \& Halpern (2015). Motivated by the fact that PSR J1023+0038 brightened by a factor of 5 at $\mathrm{GeV}$ energies when it transitioned from an RMSP to an LMXB state (Stappers et al. 2014), this candidate tMSP was found by investigating the population of unidentified Fermi gamma-ray

\footnotetext{
${ }^{14}$ Recent works by Degenaar et al. (2014) and van den Eijnden et al. (2017) indicate that dramatic type-II X-ray bursts occur due to a gap between the neutron star and the inner edge of the accretion disk.
} 
Table 1

Summary of key Observational Properties of tMSPs in RMSP and LMXB states

\begin{tabular}{|c|c|c|c|}
\hline Properties & PSR J1023+0038 & IGR J18245-2452 & XSS J12270-4859 \\
\hline \multicolumn{4}{|c|}{ Radio millisecond pulsar state } \\
\hline Steep-spectrum radio pulsations & $\checkmark$ & $\checkmark$ & $\checkmark$ \\
\hline Gamma-ray pulsations & $x$ & $\checkmark$ & $\checkmark$ \\
\hline Orbitally modulated optical and X-ray emission & $\checkmark$ & $\checkmark$ & $\checkmark$ \\
\hline Stochastic orbital variations & $\checkmark$ & $\checkmark$ & $\checkmark$ \\
\hline Radio eclipses & $\checkmark$ & $\checkmark$ & $\checkmark$ \\
\hline \multicolumn{4}{|c|}{ Low-mass X-ray binary state } \\
\hline Flat-spectrum radio emission & $\checkmark$ & $\checkmark$ & $\checkmark$ \\
\hline Gamma-ray flux enhancement & $\checkmark$ & $\checkmark$ & $\checkmark$ \\
\hline X-ray flux enhancement and pulsations & $\checkmark$ & $\checkmark$ & $\checkmark$ \\
\hline Stochastic orbital variations & $\checkmark$ & $\checkmark$ & $\checkmark$ \\
\hline
\end{tabular}

sources. More broadly, the selection criteria were based on the observed gamma-ray and multiwavelength emission from tMSPs in both RMSP and LMXB states (Patruno et al. 2014; Johnson et al. 2015). Bogdanov \& Halpern (2015) used the optical properties and X-ray light-curve phenomenology to categorize 3FGL J1544.6-1125 (also associated with X-ray source 1RXS J154439.4-112820) as a strong tMSP candidate.

More extensive studies, which analyzed the archival X-ray and radio data (Bogdanov 2016) for this source, further strengthened this claim and showed that the system had not transitioned to an RMSP state over the course of the past ten years (at least not for a prolonged period of time). Also, using spectroscopic observations, Britt et al. (2017) showed that the orbital period of 3FGL J1544.6-1125 is $\sim 0.24$ day, similar to other tMSPs $\left(P_{\text {orb }}=0.2-0.4\right.$ day $)$, and that it is situated at $3.8 \pm 0.7 \mathrm{kpc}$.

Besides PSR J1023+0038, 3FGL J1544.6-1125 at the time of its discovery was the only known (candidate) tMSP system in the LMXB state. Thus, in order to capitalize on this rare opportunity to study another tMSP in the LMXB state, we undertook a dedicated quasi-simultaneous radio/X-ray observation campaign using the VLA and the Neil Gehrels Swift Observatory X-Ray Telescope (XRT). In this paper, we present the results of this campaign. In Section 2 we present the observations and analysis. In Section 3 we present the results and in Section 4 the astrophysical implications derived from them.

\section{Very Large Array Observations}

After the discovery of 3FGL J1544.6-1125 as a strong tMSP candidate, we observed the source using the upgraded VLA (Perley et al. 2011) on four different occasions (2015 May 12, 15, 30, and June 5) under the Director's Discretionary Time project 15A-484 (PI: Jaodand; legacy ID: AJ401). Since our goal was to determine the location of 3FGL J1544.6-1125 on the radio/X-ray correlation plane, our VLA observations were dynamically scheduled to be at least quasi-simultaneous with Swift XRT observations (Figure 1). ${ }^{15}$

The duration of the scheduling block for each observation was one hour, and we used the X-band $(8-12 \mathrm{GHz})$ receiver (using the standard wide-band continuum correlator setup with 3 bit

\footnotetext{
15 The data from these observations are publicly accessible from the National Radio Astronomy Observatory (NRAO) VLA archive. https://archive.nrao. edu/archive/archiveproject.jsp.
}

sampling). The VLA was in an evolving hybrid "BnA" configuration during our observations, and offered a resolution of $\sim 1^{\prime \prime}$ at $10 \mathrm{GHz}$. In all but the third observation, some antennas were in the process of moving into or out of the BnA configuration, and so the sensitivity and $u v$-coverage were slightly compromised due to a reduced number of available antennas in the array.

In each epoch, the target source 3FGL J1544.6-1125 was observed for a total time of $\sim 32$ minutes (10 scans of $\sim 3.2$ minutes). The source $\mathrm{J} 1331+305$ (3C 286) was used as a flux calibrator and to calibrate the instrumental bandpass; calibration coefficients for the flux density scale were adopted from Perley \& Butler (2017). In all the observations, J1543-0757 was used as a gain calibrator and was visited every $\sim 4$ minutes, in between target scans. For the third and fourth epochs, a scan on the source $\mathrm{J} 1407+2827$ was also added to allow for the potential calibration of the instrumental polarization leakage. We summarize these observations in Table 2.

\subsection{VLA Data Analysis}

Every VLA scheduling block between 2013 January and 2015 September was automatically flagged and calibrated by a VLA scripted calibration pipeline. ${ }^{16}$ This pipeline uses CASA (v. 5.1.2) as the underlying software suite (for more information see McMullin et al. 2007) to handle various operations such as loading the data into a measurement set, Hanning smoothing, flux calibration, radio-frequency interference (RFI) flagging, etc.

Based on an inspection of the pipeline data products for each epoch, we identified additional RFI and antenna/frequency combinations affected by instrumental issues, and performed manual flagging to remove the affected data. Finally, we reran the pipeline to generate the best possible calibration, and applied this to the 3FGL J1544.6-1125 field before splitting the data and imaging the target field.

The brightest source in the field is located several arcminutes to the northwest of 3FGL J1544.6-1125, and has a flux density of $\sim 300 \mu \mathrm{Jy}$ (after correcting for attenuation by the primary beam). The limited brightness of this source proved insufficient to allow for the derivation of self-calibration solutions, and so no further calibration was applied to the target field.

\footnotetext{
$\overline{16}$ https://science.nrao.edu/facilities/vla/data-processing/pipeline/scriptedpipeline.
} 


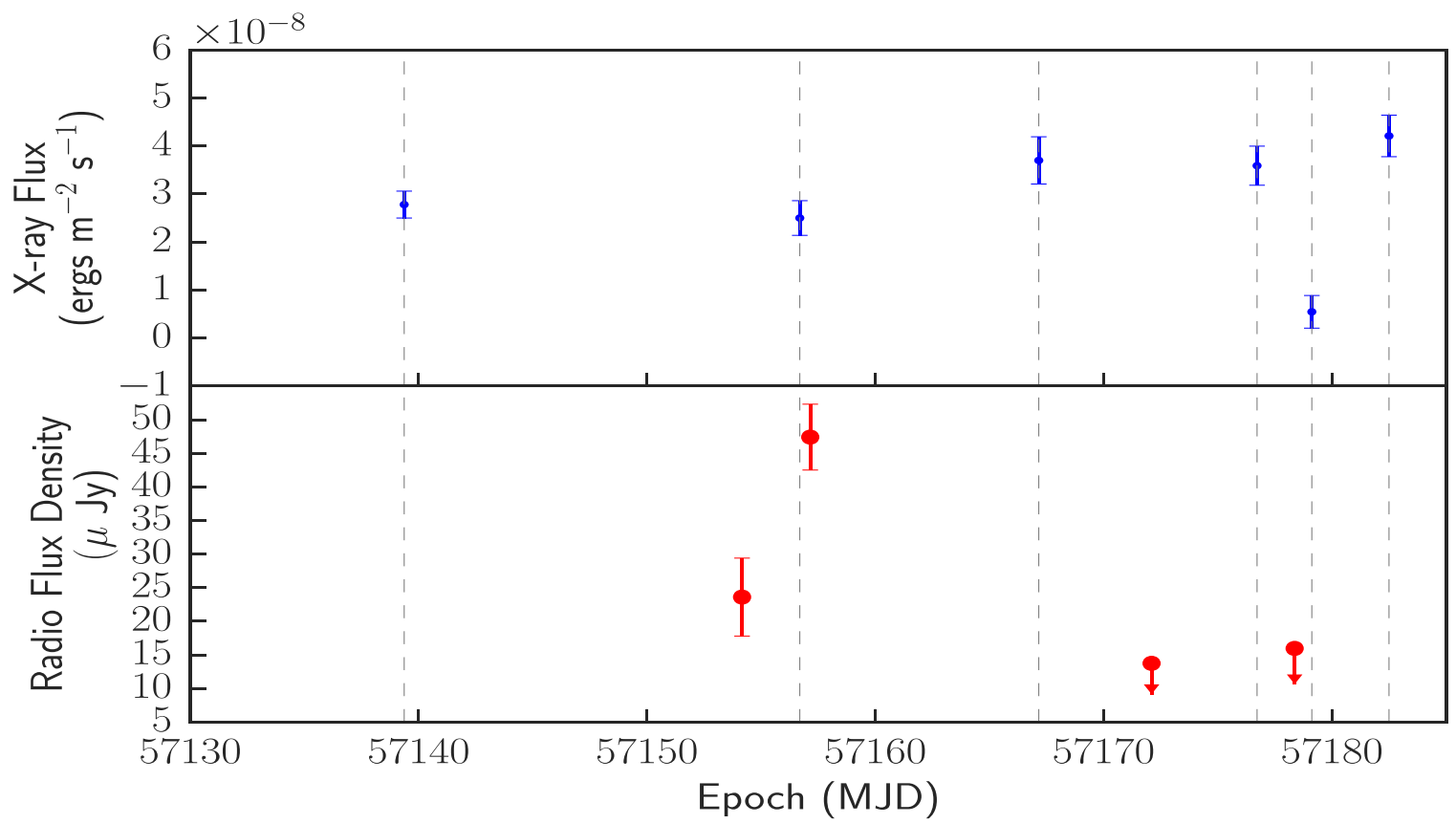

Figure 1. Radio flux density at $10 \mathrm{GHz}$ (red points; bottom panel; see Table 2) and 1-10 keV unabsorbed X-ray flux (blue points; top panel; see Table 3) as a function of modified Julian date (MJD). The Swift X-ray observations span the four-epoch VLA radio campaign, and the exact epochs of Swift observations are denoted by dashed vertical lines for reference. Note that the fifth X-ray observation shown here (at MJD 57179) was only a $418 \mathrm{~s}$ exposure, and it is possible that 3FGL J1544.6 -1125 was in the X-ray low mode for the entirety of this observation, which would explain the low measured X-ray flux compared to the other Swift observations. The upper limits $(3 \sigma)$ on VLA flux density at Epochs 3 and 4 are marked with downward arrows.

Table 2

Summary of VLA Radio Observations

\begin{tabular}{|c|c|c|c|c|c|}
\hline Epoch \# & $\begin{array}{l}\text { Obs. Date } \\
\text { (UTC) }\end{array}$ & $\begin{array}{l}\text { Obs. Start Time } \\
\text { (UTC) }\end{array}$ & Obs. Start (MJD) & $\begin{array}{l}\text { Average Flux Density } \\
(\mu \mathrm{Jy})\end{array}$ & $\begin{array}{l}\text { Uncertainty } \\
(\mu \mathrm{Jy})\end{array}$ \\
\hline 1 & $2015-05-12$ & $03: 29: 10$ & $57,154.17$ & 23.6 & 4.8 \\
\hline 2 & $2015-05-15$ & $03: 10: 26$ & $57,157.15$ & 47.7 & 6.0 \\
\hline 3 & $2015-05-30$ & $01: 56: 23$ & $57,172.10$ & $<13.8$ & $\ldots$ \\
\hline 4 & 2015-06-05 & 08:19:40 & $57,178.36$ & $<16.2$ & $\cdots$ \\
\hline
\end{tabular}

Note. Summary of VLA observations at $8-12 \mathrm{GHz}$ (X band) in BnA array configuration. At each epoch we achieved roughly 40 minutes on source and an imaging $\mathrm{rms}$ noise of $\sim 5 \mu \mathrm{Jy} /$ beam, as expected given the observational setup. 3FGL J1544.6-1125 was only convincingly detected in Epochs 1 and 2, where we used a 2D Gaussian fit to measure the flux density and quote a $1 \sigma$ uncertainty equivalent to the off-source rms noise. In Epochs 3 and 4, no significant radio emission is detected at the known source position; here we quote $3 \sigma$ upper limits based on the off-source rms noise.

For each epoch, we used the CASA task clean in interactive mode to image the full primary beam of the VLA in Stokes $I$ (total intensity), identifying and cleaning sources above $30 \mu \mathrm{Jy}$ ( $\gtrsim 5 \sigma$ compared to the noise level). We used the full bandwidth of the observations and a simple clean (natural weighting, multifrequency synthesis with a single Taylor term and a single scale).

In the resulting images, we estimated the rms noise using a source-free box near the position of 3FGL J1544.6-1125. If a peak $>3 \sigma$ was present at the known position of 3FGL J1544.6 -1125 (Bogdanov \& Halpern 2015), we used the task imfit to fit a single 2D Gaussian component (with semimajor and semiminor axes matching the size of the synthesized beam) and took the fitted value of the peak as the flux density of the point source at that epoch. For the epochs where 3FGL J1544.6 -1125 was not detected, we report a $3 \sigma$ upper limit based on the off-source rms noise. We include a $10 \%$ uncertainty to account for the absolute flux calibration of the VLA (Perley \& Butler 2017) in addition to the formal uncertainties obtained from the image rms noise, although the latter dominates.

\subsection{Swift Observations}

Prior to our VLA radio campaign, 3FGL J1544.6-1125 was already being monitored regularly, along with a few "redback" pulsars $^{17}$ and known tMSP systems, using Swift XRT (Burrows et al. 2004) in 2015. The aim of that campaign was to catch any possible transition in 3FGL J1544.6-1125 from the LMXB to RMSP state-or at least to establish whether it shows long-term X-ray brightness variations. We supplemented this campaign with an additional set of Swift XRT observations, scheduled close in time to complement the VLA observations of 3FGL J1544.6-1125.

These observations, as well as four archival observations from 2006-2012, are summarized in Table 3. We present 13 Swift observations from 2015 (each of which has a typical duration of $\sim 0.5-2 \mathrm{ks}$ ), of which six closely span the duration of the VLA observations. Although not strictly simultaneous with the latter, these cover the baseline of VLA observations in

\footnotetext{
$\overline{17}$ These are a class of eclipsing radio MSP to which all known tMSPs belong when they are in the RMSP state (see, e.g., Jaodand et al. 2017).
} 
Table 3

Summary of Swift XRT X-ray Observations

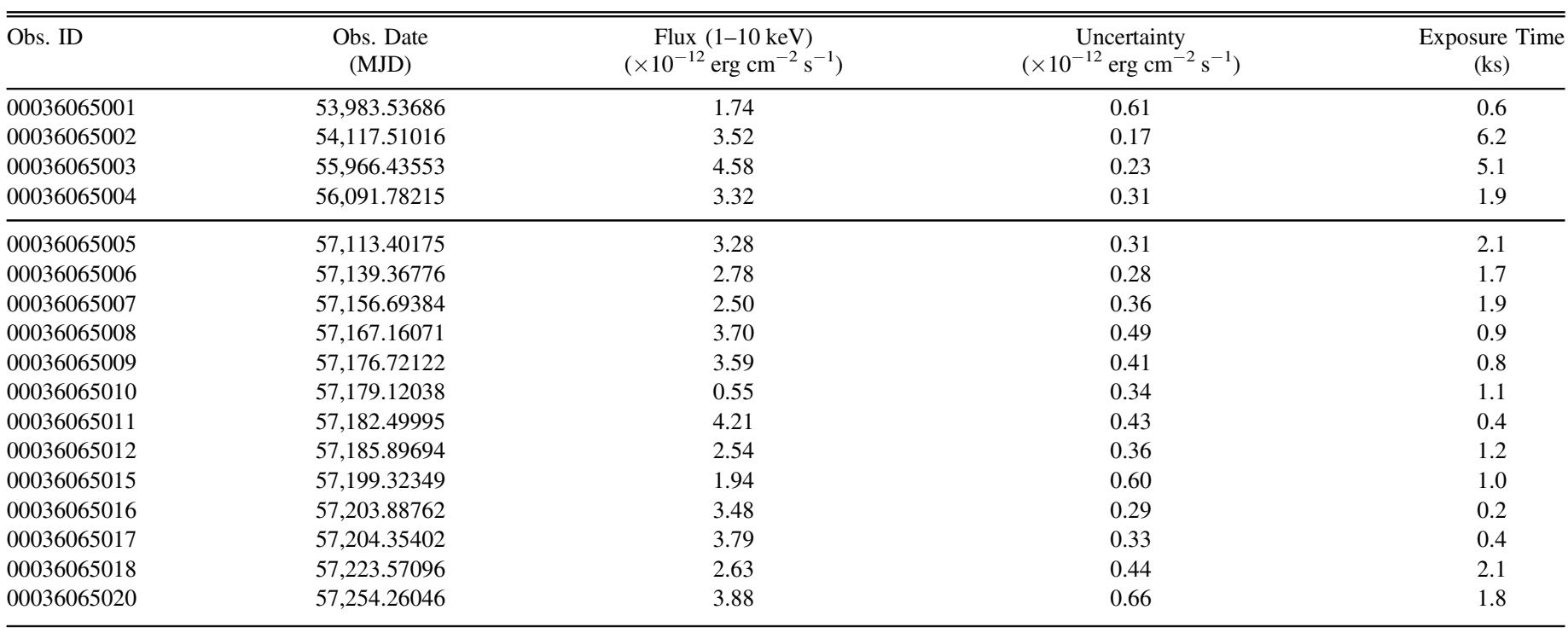

Note. The quoted fluxes are corrected for absorption; see main text. The uncertainties are at the $90 \%$ confidence level.

a quasi-simultaneous manner and demonstrate that the system was still in the LMXB state at that time (Figure 1). We note that Swift has continued to monitor this source and that other observations-in addition to those presented here, and including the UVOT instrument-also exist.

\subsection{Swift Data Analysis}

We analyzed all Swift XRT observations, taken in the photon counting mode, using the standard Swift tool xrtpipeline in HEASoft. ${ }^{18}$ Photon counts were extracted from a circular region with a radius of 20 detector pixels (corresponding to 47"). We calculated the contribution from the background by averaging counts detected in four circular regions of the same size across the field of view after verifying that they did not fall close to obvious point sources. The $1-10 \mathrm{keV}$ X-ray counts were then extracted and binned in $10 \mathrm{~s}$ intervals with the software xselect. The X-ray counts were then corrected with the task xrtlccorr, which accounts for telescope vignetting, point-spread function corrections and bad pixels/columns and rebinned the counts in intervals of $50 \mathrm{~s}$.

A summary of all the observations and associated flux measurements is presented in Table 3. The unabsorbed X-ray fluxes $(1-10 \mathrm{keV})$ for Swift XRT observations were obtained assuming a simple absorbed power-law spectral model, as measured using XMM-Newton and described in Bogdanov \& Halpern (2015). We adopted their values for photon index $\Gamma=1.68 \pm 0.04$ and a hydrogen column density of $N_{\mathrm{H}}=(1.4 \pm$ $0.1) \times 10^{21} \mathrm{~cm}^{-2}$ along the line of sight.

\section{Results}

Throughout the paper, we assume the spectroscopically estimated distance of $3.8 \pm 0.7 \mathrm{kpc}$ for 3FGL J1544.6-1125 (Britt et al. 2017).

The Swift XRT flux measurements from MJD 53,983 $-57,254$ (2006 Sep to 2015 Aug) are consistent with 3FGL J1544.6-1125 being in the LMXB state for this entire

\footnotetext{
18 Available at https://heasarc.nasa.gov/lheasoft/.
}

time. However, given that the transition to RMSP state can be rapid (e.g., Stappers et al. 2014) and might only last for months to years in some cases, we cannot rule out that 3FGL J1544.6 -1125 switched states during the sparse X-ray monitoring available before 2015. Nevertheless, it is clear that the source was in the LMXB state during our 2015 May to June VLA campaign: we find an average $\mathrm{X}$-ray luminosity of $(4.32 \pm 0.23) \times 10^{33} \mathrm{erg} \mathrm{s}^{-1}$ at this time, which we use as the point of reference for our radio measurements. While the X-ray flux here was lower on MJD 57,179, it is plausible that this observation was dominated by a single low mode in this short (418 s) observation.

\subsection{Interobservation Variability}

3FGL J1544.6-1125 was found to be faint in radio, with the strongest detection being $\sim 8 \sigma$ in significance, and two epochs only providing upper limits on the radio flux density (Table 2). Our VLA observations detect 3FGL J1544.6-1125 at 23.6土 $4.8 \mu \mathrm{Jy}$ and $47.7 \pm 6.0 \mu \mathrm{Jy}$ at Epochs 1 and 2, respectively. At Epochs 3 and 4, the source is undetected, with $3 \sigma$ upper limits of $13.8 \mu \mathrm{Jy}$ and $16.2 \mu \mathrm{Jy}$, respectively. This demonstrates a clear variability in radio brightness of a least a factor of a few on timescales of days (see Figure 2 for radio interferometric images for each of the 4 epochs). As mentioned above, strictly simultaneous radio/X-ray observations are needed to probe variability on the much shorter timescales on which 3FGL J1544.6-1125 is known to switch between X-ray low and high modes.

Given the very low average radio flux density of 3FGL J1544.6-1125, fitting for the spectral index calculated by imaging two base bands separately of radio emission from this source, or performing polarization imaging would not offer useful constraints. Indeed, the spectral index we obtain from the brightest epoch is $\alpha=0.7 \pm 1.0$, which is consistent with a flat spectrum, but with a large uncertainty. Likewise, testing for short-timescale variability is strongly limited by the source brightness and the fact that the low modes-where, in analogy to PSR J1023+0038, we expect radio brightening- 


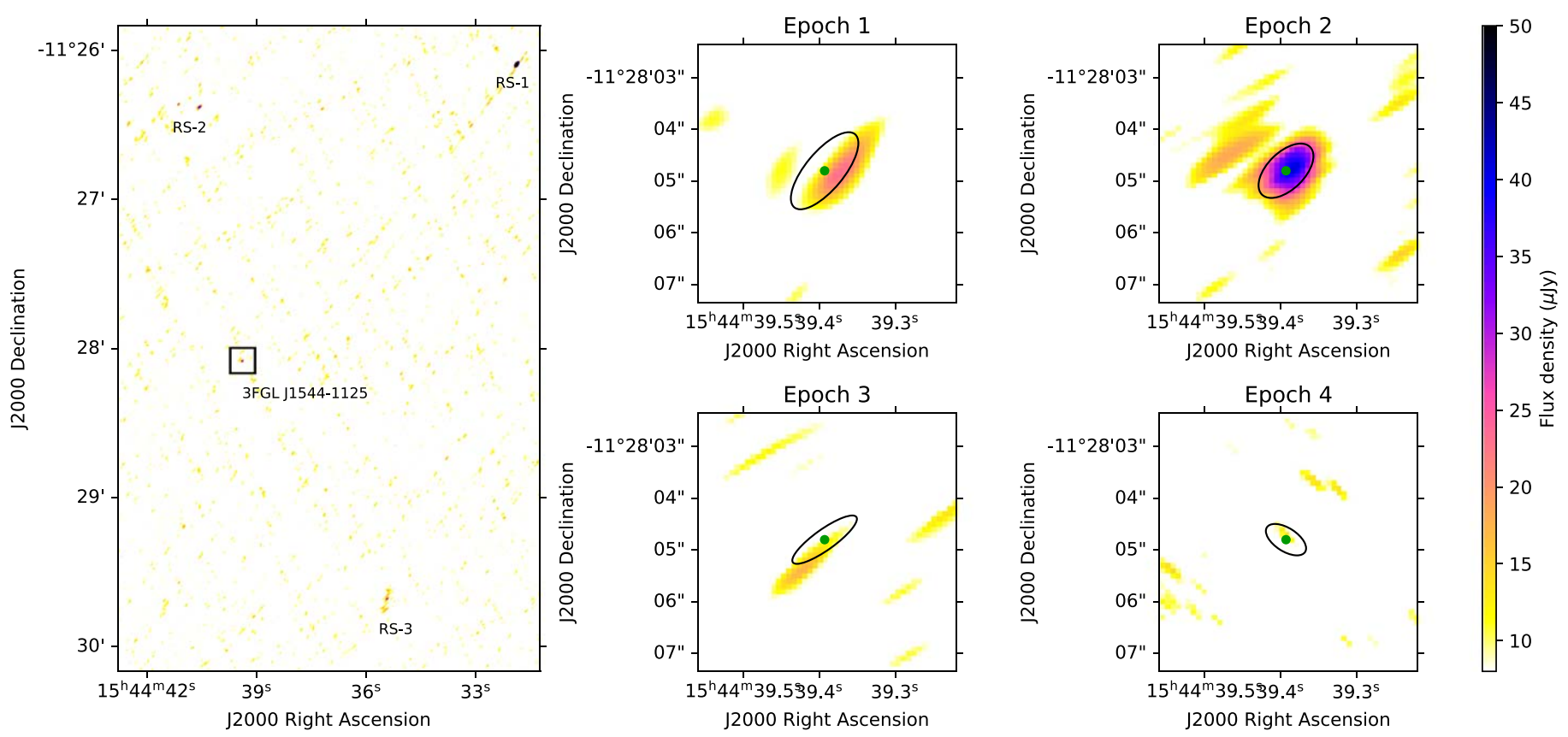

Figure 2. Radio interferometric images (Stokes $I$ ) from all four VLA observation epochs (see Table 2). Left: radio image of the second epoch showing 3FGL J1544.6 -1125 and three unidentified radio sources in the surrounding field (RS-1, RS-2, and RS-3 with the corresponding flux densities of $\sim 350 \mu \mathrm{Jy} / \mathrm{beam}, \sim 50 \mu \mathrm{Jy} / \mathrm{beam}$, and $\sim 40 \mu \mathrm{Jy} /$ beam). Right: each subpanel shows a zoomed-in radio images of 3FGL J1544.6-1125 from one of the four epochs. The synthesized beams are shown by black ellipses centered at the Gaia position of the source, which is shown by a green circle. The source is not convincingly detected during Epoch 3 or 4 .
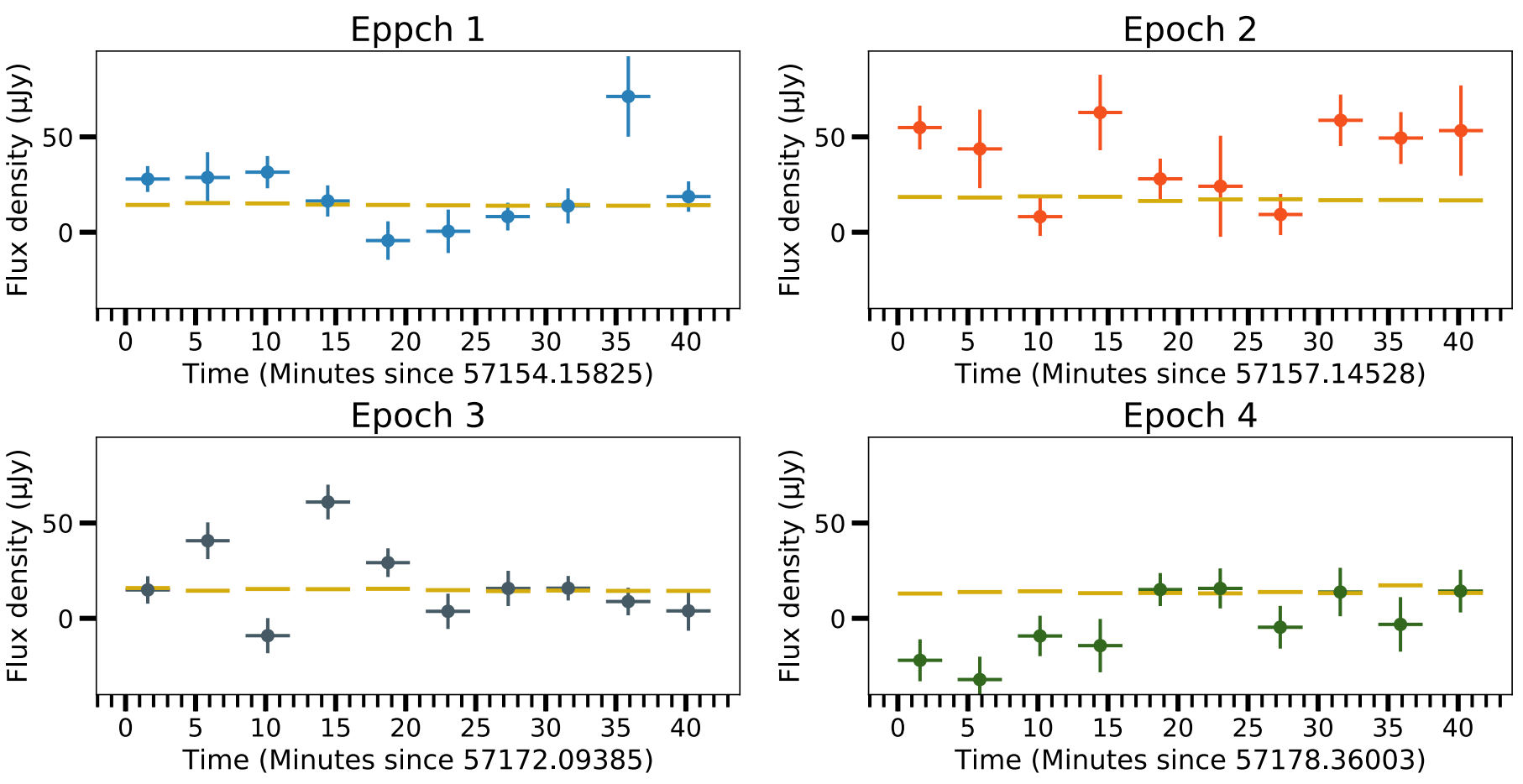

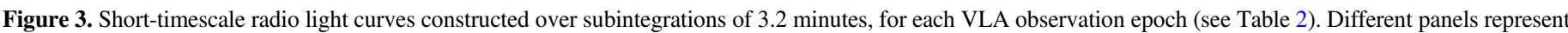

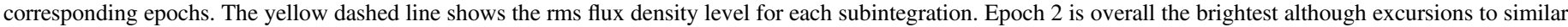
flux levels are seen for short intervals during Epoch 1 and Epoch 3.

typically last for only a few minutes to half an hour at a time (Bogdanov \& Halpern 2015). Nevertheless, we attempted to search for such variability by splitting the observation into ten $\sim 3.2$ minutes subintegrations; the light curves thus obtained are presented in Figure 3. We opted for 3.2 minutes because it is also the average scan time of the source. In all epochs, except Epoch 1, we find that the source variability is consistent with the rms noise of the image $(\sim 15 \mu \mathrm{Jy} /$ beam per subintegration). In the first epoch, we find a single bright peak of $71.2 \mu \mathrm{Jy} /$ beam in one subintegration, which is $\sim 3$ times brighter than the mean flux of the source in that epoch. However, due to the high rms noise, the significance of this bright peak is only $\sim 2 \sigma$. This hints at a potential similarity of short-term radio light-curve behavior of 3FGL J1544.6-1125 


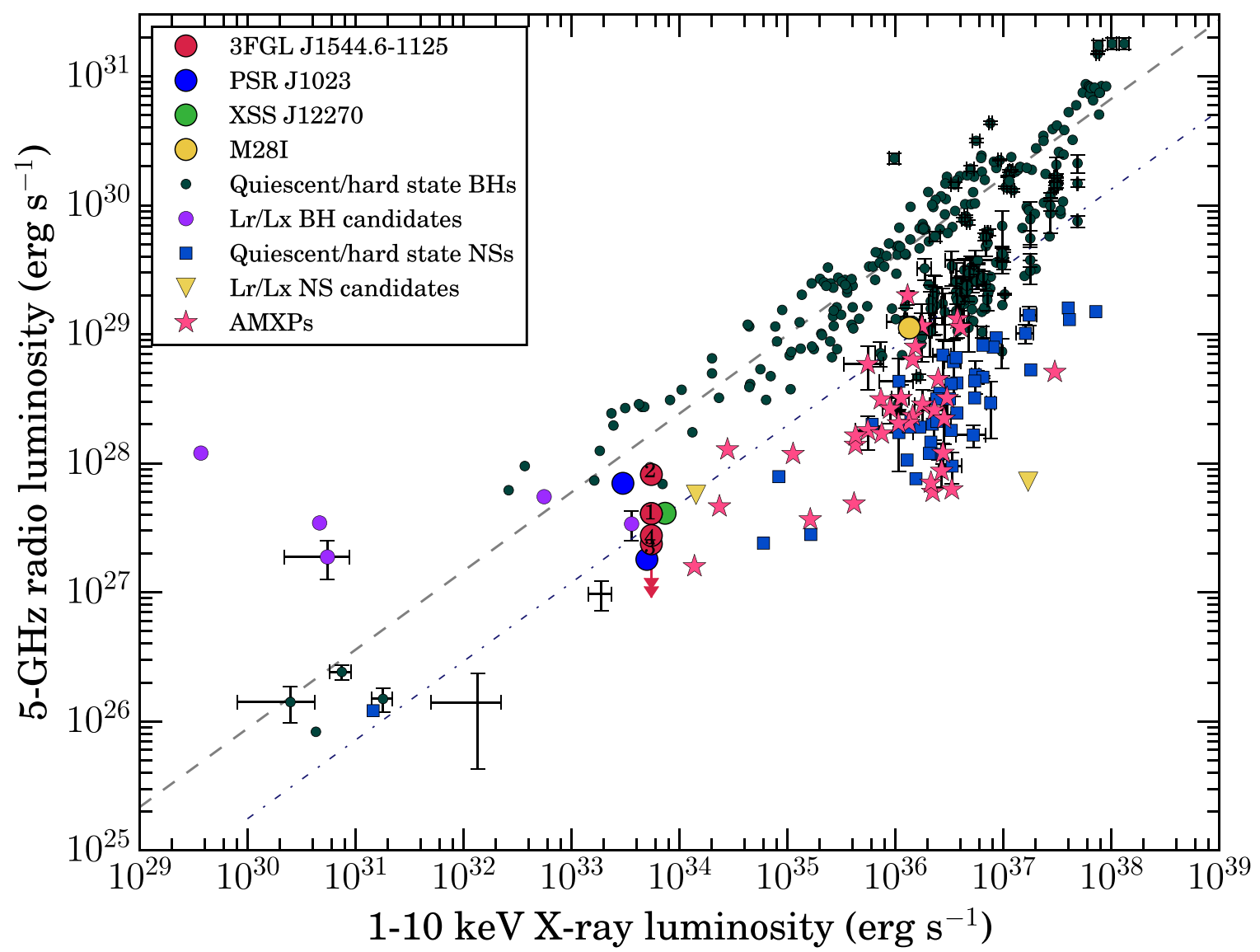

Figure 4. A phase space for NS- and BH-LMXBs, showing their $5 \mathrm{GHz}$ radio luminosities vs. their $1-10 \mathrm{keV}$ X-ray luminosities, as derived from quasi-simultaneous observations in both bands. Dark violet circles, blue squares, and pink stars represent stellar-mass black hole binaries, hard-state neutron star X-ray binaries, and accreting millisecond X-ray pulsars, respectively (the values are obtained from the database maintained by Bahramian et al. 2018). The thin gray dashed line shows the best-fit radio/X-ray luminosity correlation for BH-LMXBs (Gallo et al. 2014). The dotted-dashed blue line is the putative $L_{R} \propto L_{X}{ }^{0.7}$ scaling for tMSPs (Deller et al. 2015). Note that individual systems, with evolving accretion rates, may be represented by multiple points on this diagram. Previous radio/X-ray observations of individual tMSPs are also shown: M28I/IGR J18245-2452 (yellow circle; Papitto et al. 2013), XSS J12270-4859 (green circle; Hill et al. 2011), and PSR J1023 +0038 (blue circles; Bogdanov et al. 2018). For PSR J1023+0038, the radio/X-ray luminosities are shown separately for the low (radio brighter) and high (radio fainter) X-ray modes-as derived from strictly simultaneous radio and X-ray observations. The four radio observation epochs of 3FGL J1544.6-1125 are shown individually using red circles (see also Table 2). For all four epochs we use a median value for X-ray luminosity of $5.51 \times 10^{33} \mathrm{erg} \mathrm{s}^{-1}$. The $3 \sigma$ upper limits at Epochs 3 and 4 are indicated with an additional downward arrow.

and PSR J1023+0038. To better investigate the short-timescale $(<1 \mathrm{hr})$ variability of 3FGL J1544.6-1125 we performed (N. V. Gusinskaia et al. 2021 in preparation) a strictly simultaneous radio/X-ray observation campaign, where the $\mathrm{X}$-ray low/high-mode separation can guide the investigation of the radio light curve.

\subsection{Radio Position}

Based on the brightest radio detection (Epoch 2), we used CASA's imfit to fit a 2D Gaussian constrained to have the same dimensions as the synthesized beam, which in this case had dimensions of $2.13^{\prime \prime} \times 0.87^{\prime \prime}$ at a position angle of $143^{\circ}$ (east of north). In this way, we obtain a best-fit radio position (J2000):

R.A.: $15^{\mathrm{h}} 44^{\mathrm{m}} 39 .{ }^{\mathrm{s}} 3916 \pm 0.0082$,

decl.: $-11^{\circ} 28^{\prime} 04^{\prime \prime} 8497 \pm 0$." 1556 .

Errors as they are currently reported above are statistical from fitting the image in plane. This position is consistent to within 50 mas with the Gaia DR2 position (J2000) of the optical counterpart (i.e., the neutron star's binary companion) of R.A.: $15^{\mathrm{h}} 44^{\mathrm{m}} 39$ s.388192, decl.: $-11^{\circ} 28^{\prime} 04$." $^{\prime} 8735$ (Lindegren et al. 2018), and thus establishes a convincing association.

\subsection{Radio-X-Ray Variability}

To convert the radio luminosity to a frequency of $5 \mathrm{GHz}$, for comparison with other LMXB systems, we assume a flat spectrum $(\alpha=0)$. This is consistent with our measurement as well as with both theoretical expectations for a partially self-absorbed synchrotron jet and what is seen observationally in most LMXBs. However, note that recent work by Espinasse \& Fender (2018) shows that populations of "radio-loud" and "radio-quiet" black hole X-ray binaries differ markedly in their spectral indices. This may point to a difference in the radio emission mechanism at lower radio luminosities and require more caution in assuming a flat spectral index. While PSR J1023+0038 showed short-timescale spectral variations during its minutes-long radio flares (Bogdanov et al. 2018), averaged over longer timescales (like those probed here for 3FGL J1544.6-1125) the radio spectrum was still on average flat $(-0.3<\alpha<0.3$; Deller et al. 2015). Thus, this extrapolation should be roughly correct. We find radio luminosities of $4.08 \times 10^{27}, 8.19 \times 10^{27}, 2.37 \times 10^{27}$, and $2.76 \times 10^{27} \mathrm{erg} \mathrm{s}^{-1}$, at Epochs 1 to 4, respectively. Figure 4 is a diagram of $L_{R}$ versus $L_{X}$ showing the derived radio luminosities at each VLA observing epoch, in comparison to the average Swift X-ray luminosity quoted above. 


\section{Discussion and Conclusions}

Many of the key observational similarities of 3FGL J1544.6 -1125 compared to the well-established tMSP systems were previously presented in Bogdanov \& Halpern (2015) and Bogdanov (2016). Bogdanov \& Halpern (2015) highlight the phenomenological similarity of 3FGL J1544.6-1125's XMMNewton light curve to that of PSR J1023+0038 and XSS J12270-4859 where, during the accretion-powered state, the X-ray flux switches between quasi-stable "low" and "high" modes that are separated in X-ray luminosity by roughly an order of magnitude. Using the XMM-Newton Optical Monitor and fast photometric observations from the MDM observatory, they saw that the system showed rapid optical variability, switching within a range $\sim 0.5 \mathrm{mag}$ in a manner reminiscent of the limit-cycles seen in PSR J1023+0038. Furthermore, X-ray spectroscopy presented in Bogdanov (2016) showed that the 1-80 keV emission (from joint XMM-Newton and NuStar data) can be modeled by an absorbed power-law model with a photon index of $\Gamma \sim 1.7$. As with PSR J1023+0038, no significant spectral change was observed between the low and high X-ray modes (Bogdanov et al. 2015). This incredible similarity to PSR J1023+0038's X-ray spectroscopic behavior was also echoed by the similarity to its broadband spectral shape spanning optical, ultraviolet, and $\gamma$-rays (see Figure 4 of Bogdanov 2016).

In the work we present here, we detect $10 \mathrm{GHz}$ radio emission from 3FGL J1544.6-1125 varying in flux density from $47.7 \pm 6.0 \mu \mathrm{Jy}$ down to $\lesssim 15 \mu \mathrm{Jy}$ ( $3 \sigma$ upper limit) at four epochs spanning three weeks. During this time 3FGL J1544.6 -1125 's X-ray flux is consistent with the interpretation that it was in the persistent low-accretion-rate state seen in, e.g., tMSP PSR J1023+0038 (Bogdanov et al. 2015). Mapping 3FGL J1544.6-1125 in the radio/X-ray luminosity phase space (Figure 4) shows that it populates a similar region to the well-established tMSPs PSR J1023+0038 and XSS J12270 -4859 (Deller et al. 2015; Bogdanov et al. 2018; Hill et al. 2011). This shared phenomenology adds further support to the previous classification of 3FGL J1544.6-1125 as a strong tMSP candidate, and suggests that the tMSPs truly have quite common observational properties across the electromagnetic spectrum.

3FGL J1544.6-1125's radio flux density varies by a factor of $\gtrsim 3$ between epochs. This is consistent with the behavior of PSR J1023+0038, where the radio flux density varies by a factor of two in $\sim 2$ minutes and by an order of magnitude in $\sim 30$ minutes (see Deller et al. 2015). However, since 3FGL J1544.6-1125 is at $3.8 \pm 0.7 \mathrm{kpc}$ (Britt et al. 2017) instead of $1.368_{-0.039}^{+0.042} \mathrm{kpc}$ like PSR J1023+0038 (Deller et al. 2012), it is much more challenging to study its radio variability on such short timescales. Consequently, we plan to better investigate short-timescale $(<1 \mathrm{hr})$ variability in a strictly simultaneous radio/X-ray observation campaign, where the $\mathrm{X}$-ray low/high-mode separation can guide the investigation of the radio light curve. These observations can determine whether, like PSR J1023+0038, 3FGL J1544.6-1125's radio brightness increases during the X-ray low modes (Bogdanov et al. 2018). Given the general faintness of 3FGL J1544.6 -1125 , this may require stacking many such mode instances over several hours.

In general, our findings support the idea that tMSPs as a class are surprisingly "radio-loud" at low X-ray luminosities, when compared to expectations based on previous radio/X-ray observations of NS-LMXBs at higher accretion rates (e.g., Migliari \& Fender 2006; Migliari et al. 2011). Even comparing PSR J1023+0038 with other tMSP radio/X-ray detections, including the detection of IGR J18245-2452 in outburst (an LMXB-like outburst as seen in Papitto et al. 2013), Deller et al. (2015) tentatively postulated an empirical relation, $L_{R} \propto L_{X}{ }^{0.7}$, between radio and X-ray luminosity for tMSPs. This speculative scaling relation is, perhaps coincidentally, very similar to that suggested for some black hole (BH) LMXBs (Gallo et al. 2003; Corbel et al. 2003; Shaw et al. 2021), which show similar flat to inverted radio spectra arising from compact, self-absorbed jets (Blandford \& Königl 1979) over many orders of magnitude (Corbel et al. 2013). In the case of $\mathrm{BH}-\mathrm{LMXBs}$, the radiatively inefficient nature of the accretion flow can be attributed to the energy being liberated by infalling matter either (a) carried away in the outflow during the jetdominated regime or (b) not radiated but advected across the $\mathrm{BH}$ event horizon. However, while there may be some physical analogies to draw with $\mathrm{BH}$ jets, note that the neutron star magnetosphere and rapid rotation mean that the physical situation is likely quite different from that in tMSPs.

Placing PSR J1023+0038 in the context of other NSLMXBs, Migliari \& Fender (2006) previously established two proposed $L_{R}-L_{X}$ relations: (1) in Z-sources the radio luminosity was found to be proportional to $L_{X}^{0.66}$, whereas (2) in hard-state Atoll sources and rapid burster NS-LMXBs the radio luminosity scaled as $L_{R} \propto L_{X}^{1.4}$. The relation found for Atolltype sources remains valid over an order of magnitude in X-ray luminosity although jets being launched are less powerful than BHs at any given X-ray luminosity. They also found that after NS-LMXBs transition to the soft state their radio emission mechanism is not quenched, whereas in a recent study Gusinskaia et al. (2017) find evidence for jet quenching in an NS-LMXB. Furthermore, Migliari \& Fender (2006) highlighted that, based on the observed steeper power law for NSLMXBs, they would never enter a jet-dominated state as some BH-LMXBs do. Also, Migliari et al. (2005) theorized that millisecond pulsars in X-ray binaries, given their relatively high magnetic fields (Chakrabarty 2005), should produce jets with reduced radio luminosity.

More recent works, benefitting from a larger number of detected sources, show that the correlation for hard-state Atoll sources (index 1.4) should be viewed with skepticism given that this correlation was found over $1-1.5$ dex in $L_{X}$ (Tudor et al. 2017; Gallo et al. 2018). Indeed, the few sources tracked over more than $\sim 1-2$ dex in $L_{X}$ (mainly SAX J1808.4-3658) show significant scatter in the radio/X-ray measurements, compared to the tighter correlation observed in BH-LMXBs such as GX339-4, V404 Cyg, or XTE J1118+480. Furthermore, Tudor et al. (2017) question the very presence of single correlation. Such studies remain limited by the low number of detected NS-LMXBs, which are typically radio-fainter than BH-LMXBs by a factor of $\sim 20$ at a given $L_{X}$.

While it may be tempting to connect the radio emission seen from tMSPs in the low-level accretion state (PSR J1023+0038, XSS J12270-4859, and 3FGL J1544.6-1125) to that seen in the bright outburst of IGR J18245-2452 (see the thin dotteddashed blue line in Figure 4) we caution that these plausibly represent different accretion regimes that are not connected by a well-defined radio/X-ray scaling. Indeed, based on the anticorrelated radio/X-ray moding they observed, Bogdanov et al. (2018) conclude that PSR J1023+0038 does not fit a 
standard inflow-outflow model. Further observations of tMSPs in the intermediate X-ray luminosity range of $10^{34}-10^{36} \mathrm{erg} \mathrm{s}^{-1}$ are thus needed to better understand the similarities and differences at low and high accretion rates.

The class of accreting millisecond X-ray pulsars (AMXPs) provide an additional sample of sources that may help clarify this in the future. Just as all three known tMSPs appear like the class of "redback" MSPs when they are in the rotationpowered state, some of the AMXPs appear like tMSPs in the accretion-powered state (though none of these sources have shown radio pulsations during quiescence, e.g., Patruno et al. 2017). Tudor et al. (2017) tracked various pulsating (IGR J17511-3057，SAX J1808.4-3658, and IGR J00291 +5934) and non-pulsating (Cen X-4) NS-LMXBs in outburst and down to quiescence. They showed that, in contrast to $\mathrm{BH}$ LMXBs (which all seem to populate a radiatively inefficient track of $L_{R} \propto L_{X}^{0.7}$ ), NS-LMXBs exhibit more diverse radio/ $\mathrm{X}$-ray behaviors, possibly due to the neutron star's spin rate and magnetic field strength. For example, IGR J00291+5934 (observed at $L_{X}=10^{34}-10^{36} \mathrm{erg} \mathrm{s}^{-1}$ ) displayed a tight diskjet coupling behavior with coupling index 0.7 in the $L_{R}-L_{X}$ relationship. Conversely, SAX J1808.4-3658 showed highly variable radio emission with strong radio emission epochs consistent with X-ray reflare tails.

Moreover, the recently discovered AMXP system IGR J17591-2342 (Ferrigno et al. 2018; Sanna et al. 2018; Gusinskaia et al. 2020) was shown to be as radio-loud as BHLMXBs (Russell et al. 2018, if at $d>3 \mathrm{kpc}$ ). Thus radio emission in some AMXPs is observed to be as bright as tMSPs. However, Gallo et al. (2018) show that pulsating (AMXP) and non-pulsating NS-LMXBs can only be fitted with a common slope in the $L_{R}$ versus $L_{X}$ diagram if tMSPs are excluded from the sample. Moreover, recent exploration (van den Eijnden et al. 2021) over an ensemble of AMXPs shows that neutron stars with weaker magnetic fields are usually radio-fainter than stellar-mass black holes. These works suggest a different physical situation in the case of tMSPs accreting at a low X-ray luminosity.

Radio observations may also help in linking other X-ray binary systems to the tMSP class. For example, Degenaar et al. (2014) identified a peculiar X-ray binary, XMM J174457 -2850.3 , close to the Galactic Center. This system periodically enters accretion-dominated outbursts $\left(10^{36} \mathrm{erg} \mathrm{s}^{-1}\right)$ but at other times persists in an intermediate state where the X-ray flux is limited to $10^{33-34} \mathrm{erg} \mathrm{s}^{-1}$ for months at a time in a manner similar to tMSPs in long-term $(\sim 1 \mathrm{yr})$ low-level X-ray states. Moreover, XMM J174457-2850.3's X-ray spectrum is well fit by a power law with a photon index of 1.7. These observed properties are analogous to those of tMSPs, and Degenaar et al. (2014) invoked the interaction of the neutron star's magnetic field with the incoming accretion flow as a plausible reason for the long-term, low-level X-ray emission in such systems.

In the future, 3FGL J1544.6-1125 may eventually be detected to transition to a rotation-powered RMSP state or to enter a bright outburst at higher accretion rate, like that seen for IGR J18245-2452 (Papitto et al. 2013). Either situation could lead to the detection of the neutron star's rotation rate, via either radio or X-ray pulsations. Such a detection would establish the rotational energy budget and magnetic field strength.

If 3FGL J1544.6-1125 transitions from LMXB to RMSP state, it will be only the fourth well-established tMSP.
Currently, 3FGL J1544.6-1125 is the only (candidate) tMSP, apart from PSR J1023+0038, in the LMXB state. This makes 3FGL J1544.6-1125 a critical avenue in which to explore the nature of the tMSP accretion state and it will be important to compare it with the observations made for other transitional tMSPs in their current or previous LMXB state.

Lastly, our 3FGL J1544.6-1125 results strengthen the case that radio/X-ray/gamma-ray observations may provide a useful way of identifying new tMSP systems. Similar searches of select Fermi unassociated objects and their multiwavelength follow-ups have now yielded two other candidate tMSPs, CXOU J110926.4-650224 and 4FGL J0427.8-6704, in subluminous disk state. Hence, we are targeting unassociated Fermi gamma-ray sources using the VLA, Australia Telescope Compact Array, and Swift to look for sources whose position in the $L_{R}-L_{X}$ diagram is similar to the tMSPs'.

We thank Tom Russell for discussions about the radio analysis. A.J. and J.W.T.H. acknowledge funding from the European Research Council under the European Union's Seventh Framework Programme (FP7/2007-2013)/ERC grant agreement nr. 337062 (DRAGNET). A.J. also acknowledges support from the NuSTAR mission. A.P. acknowledges support from an NWO Vidi Fellowship. J.C.A.M.-J. is the recipient of an Australian Research Council Future Fellowship (FT 140101082). S.B. was supported in part by NASA Swift Guest Investigator Cycle 12 program grant NNX16AN79G awarded through Columbia University.

We thank B. Clark and the VLA schedulers for granting these DDT observations on short notice. The National Radio Astronomy Observatory is a facility of the National Science Foundation operated under cooperative agreement by Associated Universities, Inc. This research has made use of data and software provided by the High Energy Astrophysics Science Archive Research Center (HEASARC), which is a service of the Astrophysics Science Division at NASA/GSFC and the High Energy Astrophysics Division of the Smithsonian Astrophysical Observatory.

The data postprocessing and results preparation for this work relied heavily on Common Astronomy Software Applications (CASA 5.1) (McMullin et al. 2007). We would like to thank the staff at NRAO and externals involved in maintaining an extensive updated documentation and tutorials for CASA. This research also made use of Astropy, a community-developed core Python package for Astronomy (The Astropy Collaboration et al. 2013; Astropy Collaboration et al. 2018). Other important softwares used in this research are Matplotlib (Hunter 2007), Seaborn, and Scipy (Jones et al. 2001). Lastly, we have made extensive use of the NASA Astrophysics Data System (Eichhorn et al. 2000) and the arXiv e-print service (Ginsparg 2011).

\section{ORCID iDs}

Amruta D. Jaodand (iD https://orcid.org/0000-0002-3850-6651 Adam T. Deller (i) https://orcid.org/0000-0001-9434-3837 Jason W. T. Hessels (iD https://orcid.org/0000-00032317-1446

James C. A. Miller-Jones (ib https://orcid.org/0000-00033124-2814

Anne M. Archibald (D) https://orcid.org/0000-0003-0638-3340 Slavko Bogdanov (iD https://orcid.org/0000-0002-9870-2742 Cees Bassa (10) https://orcid.org/0000-0002-1429-9010 
Rudy Wijnands (1) https://orcid.org/0000-0002-3516-2152

Alessandro Patruno (i) https://orcid.org/0000-0002-6459-0674

\section{References}

Archibald, A. M., Kaspi, V. M., Hessels, J. W. T., et al. 2013, arXiv:1311.5161 Archibald, A. M., Stairs, I. H., Ransom, S. M., et al. 2009, Sci, 324, 1411

Archibald, A. M., Bogdanov, S., Patruno, A., et al. 2015, ApJ, 807, 62

Astropy Collaboration, Price-Whelan, A. M., Sipőcz, B. M., et al. 2018, AJ, 156, 123

Bahramian, A., Miller-Jones, J., Strader, J., et al. 2018, Radio/X-ray Correlation Database for X-ray Binaries, doi:10.5281/zenodo.1252036

Bassa, C. G., Patruno, A., Hessels, J. W. T., et al. 2014, MNRAS, 441, 1825

Blandford, R. D., \& Königl, A. 1979, ApJ, 232, 34

Bogdanov, S. 2016, ApJ, 826, 28

Bogdanov, S., \& Halpern, J. P. 2015, ApJL, 803, L27

Bogdanov, S., Archibald, A. M., Bassa, C., et al. 2015, ApJ, 806, 148

Bogdanov, S., Deller, A. T., Miller-Jones, J. C. A., et al. 2018, ApJ, 856, 54

Britt, C. T., Strader, J., Chomiuk, L., et al. 2017, ApJ, 849, 21

Burrows, D. N., Hill, J. E., Nousek, J. A., et al. 2004, Proc. SPIE, 5165, 201

Campana, S., \& Di Salvo, T. 2018, The Physics and Astrophysics of Neutron Stars, Astrophysics and Space Science Library, Vol. 457 (Berlin: Springer)

Chakrabarty, D. 2005, in ASP Conf. Ser., 328, Binary Radio Pulsars, ed. F. A. Rasio \& I. H. Stairs (San Francisco, CA: ASP), 279

Church, M. J., Gibiec, A., \& Bałucińska-Church, M. 2014, MNRAS, 438, 2784

Corbel, S., Coriat, M., Brocksopp, C., et al. 2013, MNRAS, 428, 2500

Corbel, S., Nowak, M. A., Fender, R. P., Tzioumis, A. K., \& Markoff, S. 2003, A\&A, 400, 1007

Coti Zelati, F., Papitto, A., de Martino, D., et al. 2019, A\&A, 622, A211

de Martino, D., Belloni, T., Falanga, M., et al. 2013, A\&A, 550, A89

Degenaar, N., Miller, J. M., Harrison, F. A., et al. 2014, ApJ, 796, L9

Degenaar, N., Wijnands, R., Reynolds, M. T., et al. 2014, ApJ, 792, 109

Deller, A. T., Archibald, A. M., Brisken, W. F., et al. 2012, ApJL, 756, L25

Deller, A. T., Moldon, J., Miller-Jones, J. C. A., et al. 2015, ApJ, 809, 13

Eichhorn, G., Kurtz, M. J., Accomazzi, A., Grant, C. S., \& Murray, S. S. 2000, A\&AS, 143,61

Espinasse, M., \& Fender, R. 2018, MNRAS, 473, 4122

Ferrigno, C., Bozzo, W., Sanna, A., et al. 2018, ATel, 11957, 1

Gallo, E., Degenaar, N., \& van den Eijnden, J. 2018, MNRAS, 478, L132

Gallo, E., Fender, R. P., \& Pooley, G. G. 2003, MNRAS, 344, 60

Gallo, E., Miller-Jones, J. C. A., Russell, D. M., et al. 2014, MNRAS, 445, 290 Ginsparg, P. 2011, arXiv:1108.2700

Gusinskaia, N. V., Deller, A. T., Hessels, J. W. T., et al. 2017, MNRAS, 470,1871
Gusinskaia, N. V., Russell, T. D., Hessels, J. W. T., et al. 2020, MNRAS, 492, 1091

Hasinger, G., \& van der Klis, M. 1989, A\&A, 225, 79

Hill, A. B., Szostek, A., Corbel, S., et al. 2011, MNRAS, 415, 235

Hunter, J. D. 2007, CSE, 9, 90

Jaodand, A., Archibald, A. M., Hessels, J. W. T., et al. 2016, ApJ, 830, 122

Jaodand, A., Hessels, J. W. T., \& Archibald, A. 2017, in Proc. International Astronomical Union, IAU Symp. 13 (Cambridge: Cambridge Univ. Press), 47

Johnson, T. J., Ray, P. S., Roy, J., et al. 2015, ApJ, 806, 91

Jones, E., Oliphant, T., Peterson, P., et al. 2001, SciPy: Open Source Scientific Tools for Python, http://www.scipy.org/

Kennedy, M. R., Breton, R. P., Clark, C. J., et al. 2020, MNRAS, 494, 3912

Linares, M., Bahramian, A., Heinke, C., et al. 2014, MNRAS, 438, 251

Lindegren, L., Hernández, J., Bombrun, A., et al. 2018, A\&A, 616, A2

McMullin, J. P., Waters, B., Schiebel, D., Young, W., \& Golap, K. 2007, in ASP Conf. Ser., 376, Astronomical Data Analysis Software and Systems XVI, ed. R. A. Shaw, F. Hill, \& D. J. Bell (San Francisco, CA: ASP), 127

Migliari, S., \& Fender, R. P. 2006, MNRAS, 366, 79

Migliari, S., Fender, R. P., \& van der Klis, M. 2005, MNRAS, 363, 112

Migliari, S., Miller-Jones, J. C. A., \& Russell, D. M. 2011, MNRAS, 415, 2407

Muno, M. P., Remillard, R. A., \& Chakrabarty, D. 2002, ApJ, 568, L35

Papitto, A., \& de Martino, D. 2020, arXiv:2010.09060

Papitto, A., de Martino, D., Belloni, T. M., et al. 2015, MNRAS, 453, L63

Papitto, A., Ferrigno, C., Bozzo, E., et al. 2013b, Natur, 501, 517

Papitto, A., Ambrosino, F., Stella, L., et al. 2019, ApJ, 882, 104

Parfrey, K., Spitkovsky, A., \& Beloborodov, A. M. 2016, ApJ, 822, 33

Parfrey, K., Spitkovsky, A., \& Beloborodov, A. M. 2017, MNRAS, 469, 3656

Patruno, A., Archibald, A. M., Hessels, J. W. T., et al. 2014, ApJL, 781, L3

Patruno, A., Jaodand, A., Kuiper, L., et al. 2017, ApJ, 841, 98

Perley, R. A., \& Butler, B. J. 2017, ApJS, 230, 7

Perley, R. A., Chandler, C. J., Butler, B. J., \& Wrobel, J. M. 2011, ApJL, 739, L1

Roy, J., Ray, P. S., Bhattacharyya, B., et al. 2015, ApJL, 800, L12

Russell, T. D., Degenaar, N., Wijnands, R., et al. 2018, ApJ, 869, L16

Sanna, A., Ferrigno, C., Ray, P. S., et al. 2018, A\&A, 617, L8

Shaw, A. W., Plotkin, R. M., Miller-Jones, J. C. A., et al. 2021, ApJ, 907, 34

Stappers, B. W., Archibald, A. M., Hessels, J. W. T., et al. 2014, ApJ, 790, 39

Strader, J., Li, K.-L., Chomiuk, L., et al. 2016, ApJ, 831, 89

The Astropy Collaboration, Robitaille, T. P., Tollerud, E. J., et al. 2013, A\&A, 558, A33

Tudor, V., Miller-Jones, J. C. A., Patruno, A., et al. 2017, MNRAS, 470, 324 van den Eijnden, J., Bagnoli, T., Degenaar, N., et al. 2017, MNRAS, 466, L98 van den Eijnden, J., Degenaar, N., Russell, T. D., et al. 2021, MNRAS, 507,3899 